\title{
Application of the FCM-based Neuro-fuzzy Inference System and Genetic Algorithm-polynomial Neural Network Approaches to Modelling the Thermal Conductivity of Alumina-water Nanofluids
}

\author{
M.Mehrabi, M.Sharifpur, J.P. Meyer \\ Department of Mechanical and Aeronautical Engineering, University of Pretoria, Pretoria, \\ Private Box X20, South Africa.
}

\section{ABSTRACT}

By using an FCM-based neuro-fuzzy inference system and genetic algorithm-polynomial neural network as well as experimental data, two models were established in order to predict the thermal conductivity ratio of alumina $\left(\mathrm{Al}_{2} \mathrm{O}_{3}\right)$-water nanofluids. In these models, the target parameter was the thermal conductivity ratio, and the nanoparticle volume concentration, temperature and $\mathrm{Al}_{2} \mathrm{O}_{3}$ nanoparticle size were considered as the input (design) parameters. The empirical data were divided into train and test sections for developing the models. Therefore, they were instructed by $80 \%$ of the experimental data and the remaining data (20\%) were considered for benchmarking. The results, which were obtained by the proposed FCM-based Neuro-Fuzzy Inference System (FCMANFIS) and Genetic Algorithm-Polynomial Neural Network (GA-PNN) models, were provided and discussed in detail.

Keywords: Nanofluid; Thermal conductivity ratio; FCM-based Neuro-Fuzzy Inference System (FCM-ANFIS); Genetic Algorithm-Polynomial Neural Network (GA-PNN); Group Method of Data Handling (GMDH)

Address correspondence to Prof J.P. Meyer, Department of Mechanical and Aeronautical Engineering, University of Pretoria, Private Box X20, Pretoria, 0028, South Africa, Email: josua.meyer@up.ac.za, Tel: 27(12) 420 3104, Fax: 27 (12) 4206632 


\section{INTRODUCTION}

Nanofluids are a class of heat transfer fluids which consist of a conventional base fluid such as water, engine oil, ethylene glycol (and/or mixture of them) with suspensions of low concentrations of nano-sized particles $(1-100 \mathrm{~nm})$, generally metal, metal oxide or carbon nanotubes. More heat transfer surface between particles and fluids, high dispersion stability and reduced wearing and clogging are the main advantages of nanofluids in comparison with conventional solid-liquid suspensions [1]. Over the last two decades, the study of nanofluids as potential heat transfer fluids has received significant attention, especially after Masuda et al. [2] and Choi [3] reported significant enhancement of nanofluid thermal conductivities compared with conventional working fluids.

Kleinstreuer and Feng [4] investigated the recent development of experimental and theoretical works on nanofluid thermal conductivity. They showed that most previous experimental studies focused on nanofluids containing alumina $\left(\mathrm{Al}_{2} \mathrm{O}_{3}\right)$, copper $(\mathrm{Cu})$ and copper oxide $(\mathrm{CuO})$ nanoparticles in water and ethylene glycol. Furthermore, they showed that the main focus of previous experimental works was on the study of the dependence of thermal conductivity enhancement of nanofluids on particle size, concentration and temperature.

Recently, theoretical research into information processing has increasingly been developed for use in applications. This interest was especially displayed in the development of intelligent systems, which are based on empirical data. "Norm" calculations, which are known as fuzzy logic, neural networks and genetic algorithms, are among the systems which transfer the knowledge and rules that exist beyond the 
empirical data into the network structure by their processing. Because these methods do not consider any presuppositions about statistical distribution and characteristics of the data, they are practically more efficient than common statistical methods. Many studies were conducted about the use of these approaches as effective tools for system identification. Recently, many researchers have applied these methods in order to model engineering processes. Some recent work such as Kargar et al. [5], Hojjat et al. [6] and Papari et al. [7] used a neural network approach to analyse engineering problems containing nanofluids.

In this paper we used the FCM-based Neuro-Fuzzy Inference System (FCM-ANFIS) as a method that uses neural network and fuzzy method approaches advantages for modelling the $\mathrm{Al}_{2} \mathrm{O}_{3}$-water nanofluids thermal conductivity ratio. This was done to show the high capability of this method to model engineering problems based on input output experimental data. On the other hand, due to the advantages of using evolutionary methods such as genetic algorithm to help conventional methods to perform better in the face of experimental input output data, the present ongoing research has attempted to use Genetic Algorithm-Polynomial Neural Network (GAPNN) as an evolutionary approach to model the thermal conductivity ratio of nanofluid taking into account effective parameters.

In this study, the application of these two methods is introduced for predicting the thermal conductivity ratio of $\mathrm{Al}_{2} \mathrm{O}_{3}$-water nanofluids as function of nanoparticle volume concentration, temperature and nanoparticle size. 


\section{ADAPTIVE NEURO-FUZZY INFERENCE SYSTEM}

An ANFIS system uses two neural network and fuzzy logic approaches. When these two systems are combined, they may qualitatively and quantitatively achieve a proper result that will include either fuzzy intellect or calculative abilities of a neural network. As with other fuzzy systems, the ANFIS structure is organised into two introductory and concluding parts, which are linked together by a set of rules. Five distinct layers may be recognised in the structure of an ANFIS network, which forms a multilayer network. The first layer in the ANFIS structure performs fuzzy formation and the second layer performs fuzzy "AND" and fuzzy rules. The third layer performs normalisation of membership functions and the fourth layer is the conclusive part of fuzzy rules and the last layer calculates network outputs. Detailed information about ANFIS network structure and each layer function is given in Mehrabi et al. [8].

Structure identification in fuzzy modelling involves selecting the input variables, input space partitioning, choosing the number and kinds of membership functions for inputs, creating fuzzy rules, premise and conclusion parts of fuzzy rules and selecting initial parameters for membership functions. For a given data set, different ANFIS models can be constructed using three different identification methods such as grid partitioning, subtractive clustering method and fuzzy C-means clustering [9]. In the present paper, the fuzzy C-means clustering (FCM) method is used to identify the premise membership functions for the ANFIS model.

\section{FUZZY C-MEANS CLUSTERING (FCM)}

Fuzzy C-means clustering as proposed by Bezdek [10] is a data clustering technique in which each data point belongs to two or more clusters. Fuzzy C-means is an iterative 
algorithm, which wants to find cluster centres based on minimisation of an objective function. The objective function is the sum of squares distance between each data point and the cluster centres and is weighted by its membership.

In the first step, the number of clusters $v(1 \leq v \leq n)$ and weighting exponent (fuzziness index) $m(1 \leq m<\infty)$ are randomly selected, after that the algorithm starts by initialising the cluster centres $c_{j}, j=1,2, \ldots, v$ to a random value at first time from the $n$ data points $\left\{x_{1}, x_{2}, \ldots, x_{n}\right\}$. In the next step, the membership matrix $u_{i j}=[U]$ is computed by using the following equation:

$$
u_{i j}=\frac{1}{\sum_{k=1}^{v}\left(\frac{\left\|x_{i}-c_{j}\right\|}{\left\|x_{i}-c_{k}\right\|}\right)^{\frac{2}{m-1}}}
$$

Where $\|*\|$ is any norm expressing the similarity between any measured data and the centre, so $\left\|x_{i}-c_{j}\right\|,\left\|x_{i}-c_{k}\right\|$ are the Euclidean distance between the $j$-th and $k$-th cluster centres and the $i$-th data point. In the fourth step, the objective function $J$ is computed according to Eq. 2.

$$
J\left(U, c_{1}, c_{2}, \ldots, c_{v}\right)=J_{m}=\sum_{i=1}^{n} \sum_{j=1}^{v} u_{i j}^{m} \cdot\left\|x_{i}-c_{j}\right\|^{2} \quad 1 \leq m<\infty
$$

In the final step, by using Eq. 3, the new fuzzy cluster centres $c_{j}, j=1,2, \ldots, v$ are computed [11-13].

$c_{j}=\frac{\sum_{i=1}^{n} u_{i j}^{m} \cdot x_{i}}{\sum_{i=1}^{n} u_{i j}^{m}}$ 


\section{POLYNOMIAL NEURAL NETWORK}

Polynomial neural networks are formed from the combination of linear regression method and artificial neural network. Each layer in this network is composed of a number of units (identical to neurons), which are considered as a polynomial. Polynomial networks have a pioneer structure and are formed by a number of layers. Each layer is composed of several units in which every unit is defined as a polynomial. Therefore, the parameters of this modelling method are considered as coefficients of the polynomial of units. There are various algorithms to form and instruct the polynomial networks, among which the Group Method of Data Handling (GMDH) algorithm is the most important one $[14,15]$.

The GMDH algorithm was first introduced by Ivakhnenko [16] as a learning method for modelling complex and non-linear systems. This algorithm considers many sub-models to construct and instruct polynomial networks and based on the most appropriate submodels, the final model is obtained. The GMDH training algorithm consists of two steps: in the first step, the network units are instructed, and in the second step, the best unit is selected. For these two steps, the training data are divided into two sets: the first set is used to instruct the models (to find the unit parameters) by linear regression, whereas the second set is used to compare models and select the more appropriate ones based on regularity criterion. When the best unit of a layer (a unit with minimum error) is worse than the best unit of the previous layer, the addition of layers is stopped. The best unit of the previous layer is introduced as the final output of the model and all joints that do not lead to the output unit are eliminated [17]. 


\section{GMDH polynomial neural networks}

By using a GMDH learning algorithm to train a polynomial neural network, a new class of polynomial neural network, witch is called a GMDH-type polynomial neural network, is introduced. In a GMDH-type polynomial neural network, all neurons contain an identical structure with two inputs and one output. Each neuron performs processing with five weights and one bias between input and output data. The relationship which is established between input and output variables by a GMDH-type polynomial neural network is a non-linear function as Eq. 4:

$$
z=a_{0}+\sum_{i=1}^{M} a_{i} x_{i}+\sum_{i=1}^{M} \sum_{j=1}^{M} a_{i j} x_{i} x_{j}+\sum_{i=1}^{M} \sum_{j=1}^{M} \sum_{k=1}^{M} a_{i j k} x_{i} x_{j} x_{k}+\cdots
$$

This is named a Volterra functions series. The GMDH algorithm is founded on the basis of Volterra functions series disintegration into second-rate two-variable polynomials. In fact, the algorithm objective is to find the unknown coefficients or weights of $\left[\mathbf{a}_{i, j}\right]$ in the Volterra functions series. In this manner, unknown coefficients are distributed among disintegrated factors and regulated as second-rate polynomials (Eq. 5) to specify weights and algebraic substitution of any returning factors: Volterra functions series with definite weights can be obtained from Lemke and Müller [18].

$$
f\left(x_{i}, x_{j}\right)=a_{0}+a_{1} x_{i}+a_{2} x_{j}+a_{3} x_{i}^{2}+a_{4} x_{j}^{2}+a_{5} x_{i} x_{j}
$$

\section{Genetic Optimisation of GMDH Polynomial Neural Networks}

In this paper, a genetic algorithm is applied to determine the GMDH-type polynomial neural network weights, hidden layers and bias coefficients for minimising the training 
error and to find the optimal structure for a GMDH-type polynomial neural network. The hidden layers and bias coefficients are different chromosomes that the genetic algorithm tries to find. Fig. 1 shows the combination of the three different approaches that were used to model thermal conductivity ratios in a hybrid system. By using a group method of data handling learning algorithm to instruct the polynomial neural network, the GMDH-type polynomial neural network which created the neural network part was introduced. On the other hand, the genetic algorithm was used to find the GMDH-type polynomial neural network hidden layers and bias coefficients. These three different approaches built a genetic algorithm-GMDH-type polynomial neural network hybrid system, which is called GA-PNN. The steps of this hybrid system approach are described below:

Step 1: The number of chromosome strings was selected randomly and each of them was divided into several sections. Each chromosome string was represented as a set of the connection weights (hidden layer and bias coefficients) for the GMDH-type polynomial neural network.

Step 2: For each string that was established with the training data, the fitness was measured. A string's probability of being selected for reproduction was proportional to its fitness value.

Step 3: The crossover, mutation and mating operators create the offspring that constitute the new generation. Decoding these new chromosomes, we gain a new set of weights and then submit it to the network. If the training error meets the demand, then stop.

Step 4: In the last step, the chromosome string with the smallest error in the training procedure was selected to provide the final network structure. After each run, a new set of weights was obtained and replaced with the old set. Finally, one can get a best set of 
weights (layer coefficients), and obtain a well-trained GMDH-type polynomial neural network [19-20].

\section{EFFECTIVE PARAMETERS}

There are different effective parameters on nanofluids thermal conductivity reported in literature, which can be used for modelling thermal conductivity ratios. Of these parameters we chose three important ones for this study namely particle size, volume concentration and temperature.

\section{Effect of particle size}

Chon et al. [21] measured the thermal conductivity of nanofluids containing three different sizes of alumina nanoparticles with diameters of 11,47 and $150 \mathrm{~nm}$. Their results showed that the thermal conductivity increased as particle size decreased. Li and Peterson [22] observed up to $4 \%$ positive thermal conductivity enhancement for $\mathrm{Al}_{2} \mathrm{O}_{3}$ water nanofluids containing $36 \mathrm{~nm} \mathrm{Al}_{2} \mathrm{O}_{3}$ particles compared with nanofluids containing $47 \mathrm{~nm} \mathrm{Al}_{2} \mathrm{O}_{3}$ particles at 2\% volume concentration. Patel et al. [23] measured the thermal conductivity of nanofluids containing different sizes of $\mathrm{Al}_{2} \mathrm{O}_{3}, \mathrm{CuO}$, and $\mathrm{Cu}$ in water, ethylene glycol and in transformer oil. They observed positive thermal conductivity enhancements for $\mathrm{Al}_{2} \mathrm{O}_{3}$-water with smaller nanoparticles. For $\mathrm{Al}_{2} \mathrm{O}_{3}$-water nanofluid at $2 \%$ volume concentration at $50{ }^{\circ} \mathrm{C}$, the thermal conductivity enhancement for the $11 \mathrm{~nm}$ sample $(15.5 \%)$ was approximately double the enhancement for the 150 $\mathrm{nm}(7 \%)$ sample and about 1.5 times the enhancement for the $45 \mathrm{~nm}(10.5 \%)$ sample. 


\section{Effect of volume concentration}

Most of the nanofluids thermal conductivity data in the literature exhibited a linear relationship with the particle volume concentration. However, some exceptions have showed a non-linear relationship especially at low volume concentrations [24]. In these studies, the slope of the thermal conductivity versus volume concentration can be divided into two linear regimes. At low concentrations, the slope was greater than at high concentrations. Most thermal conductivity data in the literature for $\mathrm{Al}_{2} \mathrm{O}_{3}$-water nanofluids showed that with increasing nanoparticle volume concentration, the thermal conductivity also increased [21-30], however, the intensity of the increase decreased for the larger volume concentrations.

\section{Effect of temperature}

Das et al. [25] and Putra et al. [26] measured the thermal conductivity of nanofluids containing $\mathrm{Al}_{2} \mathrm{O}_{3}$ at temperatures between 21 and $51^{\circ} \mathrm{C}$. They observed that the thermal conductivity increased as the temperature increased. Over the limited temperature range considered in their study, a gradual curve could appear linearly. Therefore, more comprehensive data are required before concluding whether the thermal conductivity exhibits a linear relationship with temperature. Chon et al. [21] reported thermal conductivity measurements of water containing $\mathrm{Al}_{2} \mathrm{O}_{3}$ nanoparticles at temperatures between 21 and $71^{\circ} \mathrm{C}$. They observed that the thermal conductivity increased as the temperature increased. However, they also experienced that at 61 and $71^{\circ} \mathrm{C}$, the trend of increasing thermal conductivity was not linear. Therefore, it can be concluded that the temperature dependence of the thermal conductivity of nanofluids is dominant. 


\section{EXPERIMENTAL DATA USED FOR TRAINING AND TESTING PROCEDURE}

Masuda et al. [2] were the first researchers who used nanoparticles for the enhancement of heat transfer in a liquid. They used the transient hot wire technique to measure the thermal conductivity ratios of $\mathrm{Al}_{2} \mathrm{O}_{3}$-water nanofluids. Their experiments included three different temperatures, which were: 32,47 and $67^{\circ} \mathrm{C}$. Lee et al. [27] experimentally investigated the thermal conductivity of $\mathrm{Al}_{2} \mathrm{O}_{3}$-water nanofluids prepared with $38.4 \mathrm{~nm}$ average diameter of alumina nanoparticle at $21^{\circ} \mathrm{C}$ temperature for four different volume concentrations (1, 2, 3 and 4\%). Wang et al. [28] measured the effective thermal conductivity of fluids and nanometer-size $\mathrm{Al}_{2} \mathrm{O}_{3}$ by using a steady-state parallel-plate technique. They dispersed $\mathrm{Al}_{2} \mathrm{O}_{3}$ powder ( $\gamma$ phase) with an average diameter of 28 into water with a vacuum pump fluid and measured the thermal conductivity ratio at $24^{\circ} \mathrm{C}$ in three different volume concentrations. Das et al. [25] investigated the thermal conductivity ratio of $\mathrm{Al}_{2} \mathrm{O}_{3}$-water nanofluids with a thermal oscillation method. They studied the temperature effect of the thermal conductivity ratio of $\mathrm{Al}_{2} \mathrm{O}_{3}$ nanoparticles with an average diameter of $38.4 \mathrm{~nm}$. Their experiments consisted of seven different temperatures $\left(21,26,31,36,41,46\right.$ and $\left.51^{\circ} \mathrm{C}\right)$ for four nanoparticle volume concentrations (1, 2, 3 and 4\%). Putra et al. [26] reported some experimental data for thermal conductivity ratio of $\mathrm{Al}_{2} \mathrm{O}_{3}$ (with an average diameter of $131.2 \mathrm{~nm}$ ) in a waterbased nanofluid over a temperature range from 21 to $51^{\circ} \mathrm{C}$ at volume concentrations of 1 and 4\%. Chon et al. [21] measured the thermal conductivity of $\mathrm{Al}_{2} \mathrm{O}_{3}$-water nanofluids in 11,47 and $150 \mathrm{~nm}$ nanoparticle sizes over a wide range of temperatures (from 21 to $71^{\circ} \mathrm{C}$ ) at 1 and $4 \%$ volume concentrations. Li and Peterson $[22,29]$ published their experimental investigation into the effect of variations in temperature and volume concentration on steady-state effective thermal conductivity of $\mathrm{Al}_{2} \mathrm{O}_{3}$-water 
suspensions. $\mathrm{Al}_{2} \mathrm{O}_{3}$ nanoparticles with 36 and $47 \mathrm{~nm}$ average diameters were blended with water at $0.5,2,4,6$ and $10 \%$ volume concentrations and the resulting suspensions were evaluated at temperatures ranging from 27.5 to $35.5^{\circ} \mathrm{C}$. Kim et al. [30] measured the thermal conductivity of alumina-water nanofluids by using the transient hot wire method. They used alumina nanoparticles with an average diameter of $38 \mathrm{~nm}$ for their work and reported the results for $0.3,0.5,0.8,1.5,2$ and $3 \%$ of volume concentrations at $25{ }^{\circ} \mathrm{C}$. Timofeeva et al. [31] investigated $\mathrm{Al}_{2} \mathrm{O}_{3}$-water nanofluids thermal conductivity for a series of nanofluids consisting of 11, 20 and $40 \mathrm{~nm}$ and volume concentrations of $2.5,5,7.5$ and $10 \%$ at $23^{\circ} \mathrm{C}$. Zhang et al. [32] used a short hot wire probe to measure the thermal conductivity ratio of $\mathrm{Al}_{2} \mathrm{O}_{3}$-water nanofluids for 10,30 and $50^{\circ} \mathrm{C}$. Ju et al. [33] reported their measurements for thermal conductivity of $\mathrm{Al}_{2} \mathrm{O}_{3}$ water suspensions with nominal diameters of 20,30 and $45 \mathrm{~nm}$ for volume concentrations up to $10 \%$. Murshed et al. [34] conducted an experimental investigation into the effective thermal conductivity of $\mathrm{Al}_{2} \mathrm{O}_{3}$ nanoparticles with average diameters of 80 and $150 \mathrm{~nm}$ in a water-based suspension. In their work, the transient hot wire technique was used to measure the thermal conductivity ratio of nanofluids at different temperatures ranging from 21 to $60^{\circ} \mathrm{C}$. Patel et al. [23] measured thermal conductivity enhancement of $\mathrm{Al}_{2} \mathrm{O}_{3}$-water nanofluids in 11,45 and $150 \mathrm{~nm}$ nanoparticle sizes for four different temperatures $\left(20,30,40\right.$ and $\left.50{ }^{\circ} \mathrm{C}\right)$ at $0.5,1,2$ and $3 \%$ volume concentrations.

In this paper, all of the above experimental data were used to model the thermal conductivity ratio $\left(\mathrm{k}_{\mathrm{eff}} / \mathrm{k}_{\mathrm{bf}}\right)$ of $\mathrm{Al}_{2} \mathrm{O}_{3}$-water nanofluids using the FCM-ANFIS and GAPNN approaches. Therefore, volume concentration $\phi$, temperature $T$ and nanoparticle size $P S$ were chosen as designing variables (input parameters) for the models. 


\section{RESULTS AND DISCUSSION}

The performance of the FCM-ANFIS and the GA-PNN proposed models was tested with the sum of the squares due to the error or summed squares of residuals $(S S E)$ and root mean square errors $(R M S E)$. If $Q_{1}, Q_{2}, Q_{3}, \ldots, Q_{n}$ are $n$ observed values, $P_{1}, P_{2}$, $P_{3}, \ldots, P_{n}$ are $n$ predicted values, then $S S E$ and $R M S E$ values are as follows:

$$
\begin{aligned}
& S S E=\sum_{i=1}^{n}\left(Q_{i}-P_{i}\right)^{2} \\
& R M S E=\sqrt{\frac{1}{n} \cdot \sum_{i=1}^{n}\left(Q_{i}-P_{i}\right)^{2}}
\end{aligned}
$$

A total of 125 input output experimental data points obtained from literature were used for three effective input parameters (volume concentration $\phi$, temperature $T$ and nanoparticle size $P S$ ). These data were divided into two subsets as $80 \%$ for training and $20 \%$ for testing purposes. The characterisation of the FCM-ANFIS model is shown in Table 1. The structure of the GA-PNN model is shown in Fig. 2 corresponding to the

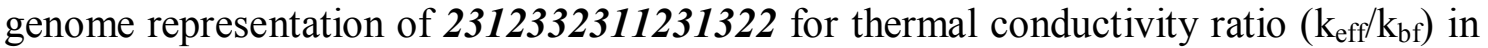
which 1,2 and 3 stand for volume concentration $\phi(\%)$, temperature $T\left({ }^{\circ} \mathrm{C}\right)$ and nanoparticle size $P S(\mathrm{~nm})$, respectively. The corresponding polynomial representation of models for $\mathrm{k}_{\mathrm{eff}} / \mathrm{k}_{\mathrm{bf}}$ is shown in the appendix.

Two statistical criteria which were mentioned before, were used to determine how well the FCM-ANFIS and GA-PNN models could predict the thermal conductivity ratio $\mathrm{k}_{\mathrm{eff}} / \mathrm{k}_{\mathrm{bf}}$ of $\mathrm{Al}_{2} \mathrm{O}_{3}$-water nanofluids corresponding to various values of inlet variables. Figures 3-7 show plots comparing the experimental data, FCM-ANFIS and GA-PNN models. These diagrams demonstrate that the predicted values are close to the 
experimental data, as many of the modelled data points fall very close to the experimental value.

Fig. 3 shows the experimental results of Lee et al. [27] compared with the FCM-ANFIS and the GA-PNN models for a particle size of $38.4 \mathrm{~nm}$, temperature of $21^{\circ} \mathrm{C}$ at four different volume concentrations. The GA-PNN model is in very good agreement with the experimental data $\left(S S E=1.527 \times 10^{-5}\right.$ and $\left.R M S E=0.002\right)$. Therefore, the GAPNN model is well matched with the experimental data. The FCM-ANFIS (SSE $=2.237 \times 10^{-6}$ and $\left.R M S E=0.0011\right)$ model is not as good as the GA-PNN model. Although the FCM-ANFIS model is not well matched with the experimental data, the maximum relative error is less than $2.5 \%$.

Fig. 4 shows the experimental results of Putra et al. [26] compared with the FCMANFIS and the GA-PNN models for a particle size of $131.2 \mathrm{~nm}$, volume concentration of $1 \%$ and seven different temperatures. The GA-PNN model is in good agreement with the experimental data $\left(S S E=1.887 \times 10^{-4}\right.$ and $\left.R M S E=0.0061\right)$, the difference between the model outputs and experimental results is less than 4\%. The FCM-ANFIS model is in very good agreement with experimental results at higher temperatures from $36-51^{\circ} \mathrm{C}\left(S S E=2.006 \times 10^{-5}\right.$ and $\left.R M S E=0.002\right)$ but for temperatures between 21 $36^{\circ} \mathrm{C}$ the FCM-ANFIS model is not as good as the higher temperatures. However, the maximum difference between the experimental data and the FCM-ANFIS model at the lowest temperature of $21^{\circ} \mathrm{C}$ is about $3 \%$.

In Fig. 5, the experimental results of $\mathrm{Li}$ and Peterson [22] compared with the FCMANFIS and the GA-PNN models are presented for a particle size of $36 \mathrm{~nm}$, temperature of $30.5^{\circ} \mathrm{C}$ at four different volume concentrations. The FCM-ANFIS model (SSE 
$=3.582 \times 10^{-5}$ and $\left.R M S E=0.0004\right)$ is well matched and the GA-PNN model $(S S E$ $=6.132 \times 10^{-4}$ and $\left.R M S E=0.0018\right)$ is also in good agreement with the experimental data. For the FCM-ANFIS model at $\phi=2 \%$ and $\phi=6 \%$, the model is approximately the same as the experimental data.

Fig. 6 shows the experimental results of Kim et al. [30] compared with the FCMANFIS and the GA-PNN models for a particle size of $38 \mathrm{~nm}$, temperature of $25{ }^{\circ} \mathrm{C}$ at five different volume concentrations. The FCM-ANFIS model $\left(S S E=8.39 \times 10^{-5}\right.$ and $R M S E=0.0046)$ and the GA-PNN model $\left(S S E=3.576 \times 10^{-5}\right.$ and $\left.R M S E=0.0029\right)$ are in good agreement with the experimental data. However, as the volume concentration increases, the accuracy of the FCM-ANFIS is better than that of the GA-PNN model.

In Fig. 7, the experimental results of Patal et al. [23] is compared with those of the FCM-ANFIS and the GA-PNN models for a particle size of $150 \mathrm{~nm}$, volume concentration of $2 \%$ at four different temperatures. The FCM-ANFIS model matches the data very well $\left(S S E=3.179 \times 10^{-4}\right.$ and $\left.R M S E=0.0126\right)$. However, the GA-PNN model $(S S E=0.0026$ and $R M S E=0.0361)$ is not in such a good agreement with the experimental data and the difference between this model and GA-PNN output for $T=50^{\circ} \mathrm{C}$ is about $6 \%$. 


\section{CONCLUSIONS}

This study showed the high capability of artificial intelligent methods for modelling engineering problems containing nanofluids based on input output experimental data, which were published in literature, for this purpose, the FCM-ANFIS and the GA-PNN approaches were developed for modelling the thermal conductivity ratio of $\mathrm{Al}_{2} \mathrm{O}_{3}$-water nanofluids as function of particle size, temperature and volume concentration.

In the FCM-ANFIS method, which consists of a neural network combined with a fuzzy logic approach, the fuzzy C-means clustering is used as an identification method. The Adaptive Neuro-Fuzzy Inference System (ANFIS) uses neural network and fuzzy logic approaches at the same time to combine the advantages of each method to achieve a better performance. In the GA-PNN hybrid system, which consists of neural network and genetic algorithm parts, the genetic algorithm is used to find the best network weights for minimising the training error and finding the optimal structure for a GMDH-type polynomial neural network. In the neural network part of this hybrid system the Group Method of Data Handling (GMDH) learning approach is used to learn a second rate polynomial neural network.

After a literature review of experimental works on $\mathrm{Al}_{2} \mathrm{O}_{3}$-water nanofluids thermal conductivity, we chose particle size, temperature and volume concentration as most effective parameters of thermal conductivity ratio. After choosing these effective (input) parameters, we used 125 input output experimental data points obtained from literature to model the thermal conductivity ratio by using the FCM-ANFIS and GA-PNN approaches. The characterisation of the FCM-ANFIS model with detailed information is mentioned in a table. The structure of the GA-PNN model based on the genome 
representation for thermal conductivity ratio with respect to effective (input) parameters is shown in a figure.

The result statistical error analyses review shows that our proposed models are in good agreement compared with experimental data. Although the FCM-ANFIS approach shows better agreement with experimental data in comparison with the GA-PNN method, the differences between the GA-PNN model outputs and experimental results in the worst-case scenario are about $6 \%$.

\section{REFERENCES}

[1] R. Saidur, K.Y. Leong, H.A. Mohammad, A review on applications and challenges of nanofluids, Renewable and Sustainable Energy Reviews 15 (2011) 1646-1668.

[2] H. Masuda, A. Ebata, K. Teramae, N. Hishinuma, Alteration of thermal conductivity and viscosity of liquid by dispersing ultra-fine particles (dispersion of c- $\mathrm{Al}_{2} \mathrm{O}_{3}, \mathrm{SiO}_{2}$ and $\mathrm{TiO}_{2}$ ultra-fine particles), Netsu Bussei 4 (1993) 227-233.

[3] S.U.S. Choi, Enhancing thermal conductivity of fluids with nanoparticles. ASME FED 231 (1995) 99-103.

[4] C. Kleinstreuer, Y. Feng, Experimental and theoretical studies of nanofluid thermal conductivity enhancement: a review, Nanoscale Research Letters 6 (2011) 229.

[5] A. Kargar, B. Ghasemi, S.M. Aminossadati, An artificial neural network approach to cooling analysis of electronic components in enclosures filled with nanofluids, Journal of Electronic Packaging, 131(2011) 011010-1. 
[6] M. Hojjat, S.Gh. Etemad, R. Bagheri, J. Thibault, Thermal conductivity of nonNewtonian nanofluids: Experimental data and modeling using neural network, International Journal of Heat and Mass Transfer 54 (2011) 1017-1023.

[7] M.M. Papari, F.Yousefi, J. Moghadasi, H. Karimi, A. Campo, Modeling thermal conductivity augmentation of nanofluids using diffusion neural networks, International Journal of Thermal Sciences 50 (2011) 44-52.

[8] M. Mehrabi, S.M. Pesteei, T. Pashaee G., Modeling of heat transfer and fluid flow characteristics of helicoidal double-pipe heat exchangers using Adaptive Neuro-Fuzzy Inference System (ANFIS), International Communications in Heat and Mass Transfer 38 (4) (2011) 525-532.

[9] H. Jalalifar, S. Mojedifar, A.A. Sahebi, H. Nezamabadi-pour, Application of the adaptive neuro-fuzzy inference system for prediction of a rock engineering classification system, Computers and Geotechnics 38 (2011) 783-790.

[10] J.C. Bezdek, Fuzzy Mathematics in Pattern Classification. PhD dissertation, Cornell University, Ithaca, NY, 1973.

[11] S.S. Kim, D.J. Lee, K.C. Kwak, J.H. Park, J.W. Ryu, Speech recognition using integra-normalizer and neuro-fuzzy method, Conference Record of the Asilomar Conference on Signals, Systems and Computers 2 (2000) 1498-1501.

[12] M.F. Othman, T. Moh, Neuro Fuzzy Classification and Detection Technique for Bioinformatics Problems, Proceedings of the First Asia International Conference on Modelling \& Simulation (AMS'07) 0-7695-2845-7/07 375-380. 
[13] S. Ibrahim, N.E.A. Khalid, M. Manaf, Seed-Based Region Growing (SBRG) vs Adaptive Network-Based Inference System (ANFIS) vs Fuzzy c-Means (FCM): Brain Abnormalities Segmentation, Proceedings of World Academy of Science, Engineering and Technology 68 (2010) 425-435.

[14] A.G. Ivakhnenko, Polynomial Theory of Complex Systems, IEEE Transaction on Systems, Man and Cybernetics SMC-1 (4) (1971) 364-378.

[15] A.G. Ivakhenko, G.A. Ivakhenko, the Review of Problems Solvable by Algorithms of Group Method of Data Handling, Pattern Recognition and Image Analysis 5(4) (1995) 527-535.

[16] A.G. Ivakhnenko, the Group Method of Data Handling - A Rival of the Method of Stochastic Approximation, Soviet Automatic Control 13 (3) (1966) 43-55.

[17] K. Fujimoto, S. Nakabayashi, Applying GMDH algorithm to extract rules from examples, Systems Analysis Modelling Simulation 43 (10) (2003) 1311-1319.

[18] F. Lemke, J.A. Müller, Self-organizing data mining, Systems analysis modelling simulation 43(2) (2003) 231-240.

[19] M. Saemi, M. Ahmadi, A. Yazdian Varjani, Design of neural networks using genetic algorithm for the permeability estimation of the reservoir, Journal of Petroleum Science and Engineering 59 (2007) 97-105.

[20] N.Y. Nikolaev, H. Iba, Learning polynomial feedforward neural networks by genetic programming and backpropagation, IEEE Transactions on Neural Networks, 14(2) (2003) 337-350. 
[21] C.H. Chon, K.D. Kihm, S.P. Lee, S.U.S. Choi, Empirical correlation finding the role of temperature and particle size for nanofluid $\left(\mathrm{Al}_{2} \mathrm{O}_{3}\right)$ thermal conductivity enhancement, Applied Physics Letters 87 (2005) 153107.

[22] C.H. Li, G.P. Peterson, The effect of particle size on the effective thermal conductivity of $\mathrm{Al}_{2} \mathrm{O}_{3}$-water nanofluids, Journal of Applied Physics 101 (2007) 044312.

[23] H.E. Patel, T. Sundararajan, S.K. Das, An experimental investigation into the thermal conductivity enhancement in oxide and metallic nanofluids, Journal of Nanoparticle Research 12 (2010) 1015-1031.

[24] S.M.S. Murshed, K.C. Leong, C. Yang, Enhanced thermal conductivity of $\mathrm{TiO}_{2}$ water based nanofluids. International Journal of Thermal Sciences 44(4) (2005) 367373.

[25] S.K. Das, N. Putra, P. Thiesen, W. Roetzel, Temperature dependence of thermal conductivity enhancement for nanofluids, Journal of Heat Transfer 125 (2003) 567574.

[26] N. Putra, W. Roetzel, S.K. Das, Natural convection of nano-fluids, Heat and Mass Transfer 39 (2003) 775-784.

[27] S. Lee, S.U.S. Choi, S. Li, J.A. Eastman, Measuring thermal conductivity of fluids containing oxide nanoparticles, Journal of Heat Transfer 121(2) (1999) 280-289.

[28] X. Wang, X. Xu, S.U.S. Choi, Thermal conductivity of nanoparticle-fluid mixture, Journal of Thermophysics and Heat Transfer 13(4) (1999) 474-480. 
[29] C.H. Li, G.P. Peterson, Experimental investigation of temperature and volume fraction variations on the effective thermal conductivity of nanoparticle suspensions (nanofluids), Journal of Applied Physics 99 (2006) 084314.

[30] S.H. Kim, S.R. Choi, D. Kim, Thermal conductivity of metal-oxide nanofluids: particle size dependence and effect of laser irradiation, Journal of Heat Transfer 129 (2007) 298-307.

[31] E.V. Timofeeva, A.N. Gavrilov, J.M. McCloskey, Y.V. Tolmachev, Thermal conductivity and particle agglomeration in alumina nanofluids: experiment and theory, Physical Review E 76 (2007) 061203.

[32] X. Zhang, H. Gu, M. Fujii, Effective thermal conductivity and thermal diffusivity of nanofluids containing spherical and cylindrical nanoparticles, Experimental Thermal and Fluid Science 31 (2007) 593-599.

[33] Y.S. Ju, J. Kim, M.T Hung, Experimental Study of Heat Conduction in Aqueous Suspensions of Aluminium Oxide Nanoparticles, Journal of Heat Transfer 130 (2008) 092403-1.

[34] S.M.S. Murshed, K.C. Leong, C. Yang, Investigations of thermal conductivity and viscosity of nanofluids, International Journal of Thermal Sciences 47 (2008) 560-568. 


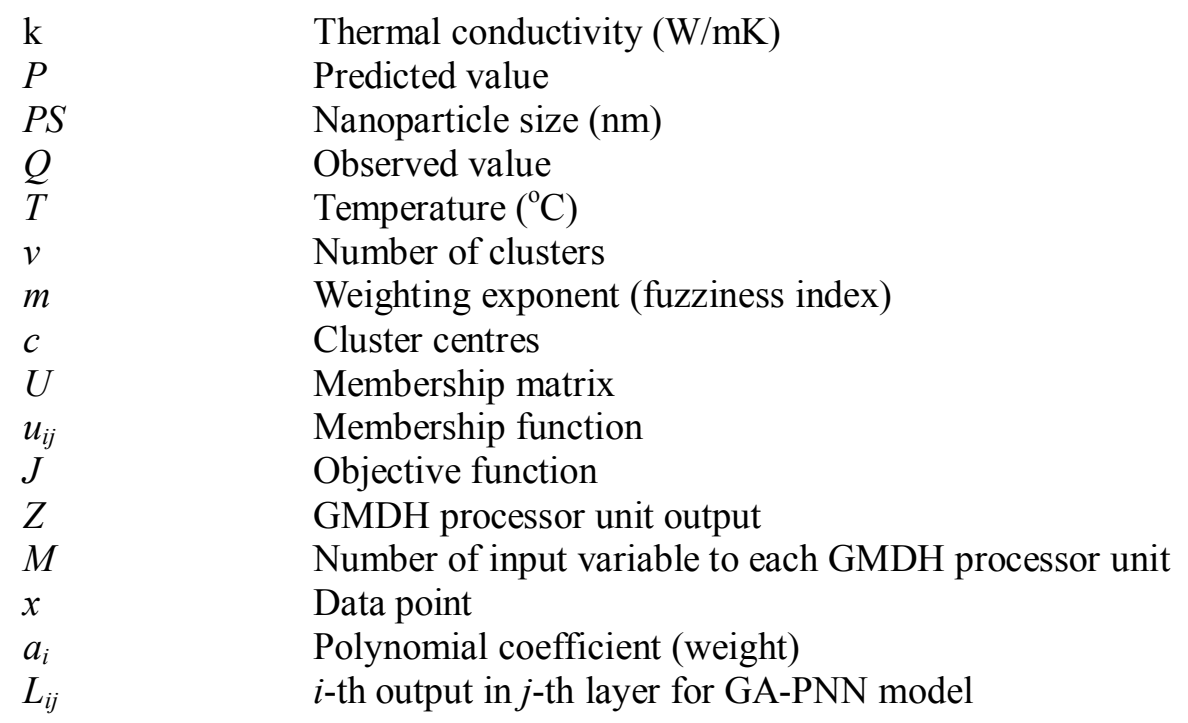

Greek letters

$\phi$

Volume concentration (\%)

Subscripts

eff

bf

Effective

Base fluid

Abbreviation

FCM-ANFIS

GA-PNN

GMDH

FCM-based Neuro-Fuzzy Inference System

$S S E$

RMSE

Genetic Algorithm-GMDH Polynomial Neural Network

Group Method of Data Handling

Sum of squares due to error or summed square of residuals

Root mean square error 


\section{Appendix:}

$$
\begin{aligned}
& L_{11}=a_{1,0}+a_{1,1} \cdot T+a_{1,2} \cdot P S+a_{1,3} \cdot T \cdot P S+a_{1,4} \cdot T^{2}+a_{1,5} \cdot P S^{2} \\
& L_{21}=a_{2,0}+a_{2,1} \cdot \phi+a_{2,2} \cdot T+a_{2,3} \cdot \phi \cdot T+a_{2,4} \cdot \phi^{2}+a_{2,5} \cdot T^{2} \\
& L_{31}=a_{3,0}+a_{3,1} \cdot \phi+a_{3,2} \cdot P S+a_{3,3} \cdot \phi \cdot P S+a_{3,4} \cdot \phi^{2}+a_{3,5} \cdot P S^{2} \\
& L_{12}=a_{4,0}+a_{4,1} \cdot L_{11}+a_{4,2} \cdot L_{21}+a_{4,3} \cdot L_{11} \cdot L_{21}+a_{4,4} \cdot L_{11}^{2}+a_{4,5} \cdot L_{21}^{2} \\
& L_{22}=a_{5,0}+a_{5,1} \cdot P S+a_{5,2} \cdot L_{11}+a_{5,3} \cdot P S \cdot L_{11}+a_{5,4} \cdot P S^{2}+a_{5,5} \cdot L_{11}{ }^{2} \\
& L_{32}=a_{6,0}+a_{6,1} \cdot \phi+a_{6,2} \cdot L_{11}+a_{6,3} \cdot \phi \cdot L_{11}+a_{6,4} \cdot \phi^{2}+a_{6,5} \cdot L_{11}^{2} \\
& L_{42}=a_{7,0}+a_{7,1} \cdot L_{31}+a_{7,2} \cdot T+a_{7,3} \cdot L_{31} \cdot T+a_{7,4} \cdot L_{31}^{2}+a_{7,5} \cdot T^{2} \\
& L_{13}=a_{8,0}+a_{8,1} \cdot L_{12}+a_{8,2} \cdot L_{22}+a_{8,3} \cdot L_{12} \cdot L_{22}+a_{8,4} \cdot L_{12}{ }^{2}+a_{8,5} \cdot L_{22}{ }^{2} \\
& L_{23}=a_{9,0}+a_{9,1} \cdot L_{32}+a_{9,2} \cdot L_{42}+a_{9,3} \cdot L_{32} \cdot L_{42}+a_{9,4} \cdot L_{32}{ }^{2}+a_{9,5} \cdot L_{42}{ }^{2} \\
& \frac{k_{e f f}}{k_{b f}}=a_{10,0}+a_{10,1} \cdot L_{13}+a_{10,2} \cdot L_{23}+a_{10,3} \cdot L_{13} \cdot L_{23}+a_{10,4} \cdot L_{13}{ }^{2}+a_{10,5} \cdot L_{23}{ }^{2}
\end{aligned}
$$

$\left[\mathbf{a}_{i, j}\right]=\left[\begin{array}{cccccc}1.0735962 & -0.0020244 & 0.0605055 & 8.35 \times 10^{-6} & -0.0064905 & 0.0001505 \\ 0.8718401 & 0.0098069 & 0.0011233 & -7.83 \times 10^{-5} & -7.14 \times 10^{-6} & -7.75 \times 10^{-6} \\ 0.8733007 & 0.0069859 & 0.0177932 & -7.08 \times 10^{-5} & -2.67 \times 10^{-3} & 7.96 \times 10^{-4} \\ 10.668079 & -15.644669 & -3.3349184 & 3.9400101 & -1.6277918 & 7.0065401 \\ -2.6847113 & -0.3084822 & 6.3004023 & -1.26 \times 10^{-3} & -2.5900101 & 0.2760002 \\ 3.4734893 & -0.0050944 & -5.2272048 & -9.32 \times 10^{-5} & 2.5841052 & 0.0140529 \\ 0.4093305 & 0.4501285 & -0.0042521 & 0.1989611 & 6.83 \times 10^{-6} & 0.0025185 \\ 0.9721418 & -2.0553909 & 1.2665332 & -2.3233686 & -4.1070767 & 7.2531441 \\ 0.2394494 & 2.1513521 & -1.5733885 & 13.480884 & 15.199995 & -28.498592 \\ -0.0477731 & 1.0660376 & -0.0012041 & 0.0495427 & 0.7246142 & -0.7942074\end{array}\right]$


Table 1

Different parameter types and their values used for training FCM-ANFIS

\begin{tabular}{|l|l|}
\hline Parameters & FCM-ANFIS \\
\hline Mumbership functions type & Gaussian \\
Output membership functions & Linear \\
Number of nodes & 54 \\
Number of linear parameters & 24 \\
Number of non-linear parameters & 36 \\
Number of training data pairs & 125 \\
Number of fuzzy rules & 6 \\
\hline
\end{tabular}




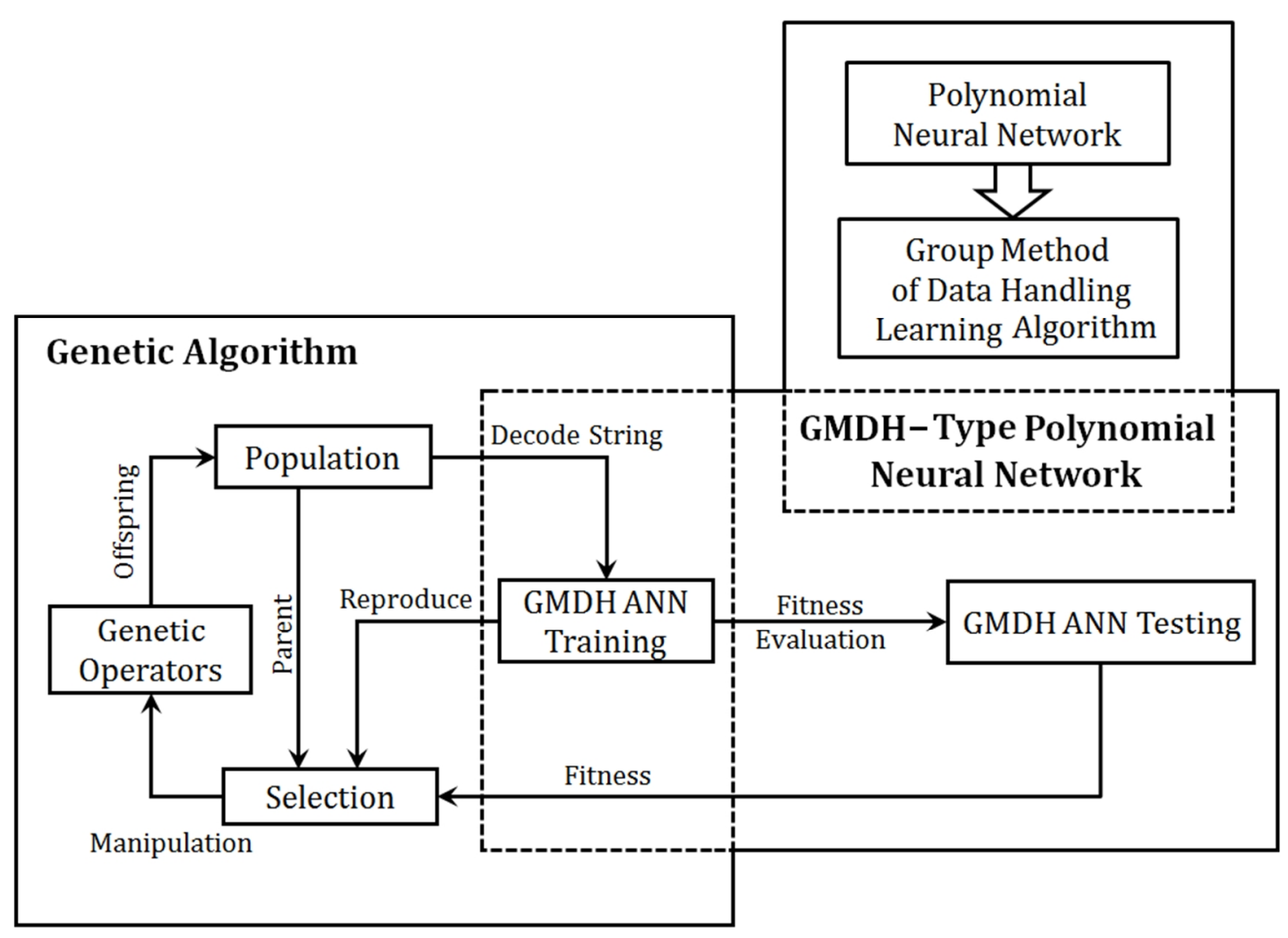

Fig.1 Combination of genetic algorithm and GMDH-type polynomial neural network approaches 


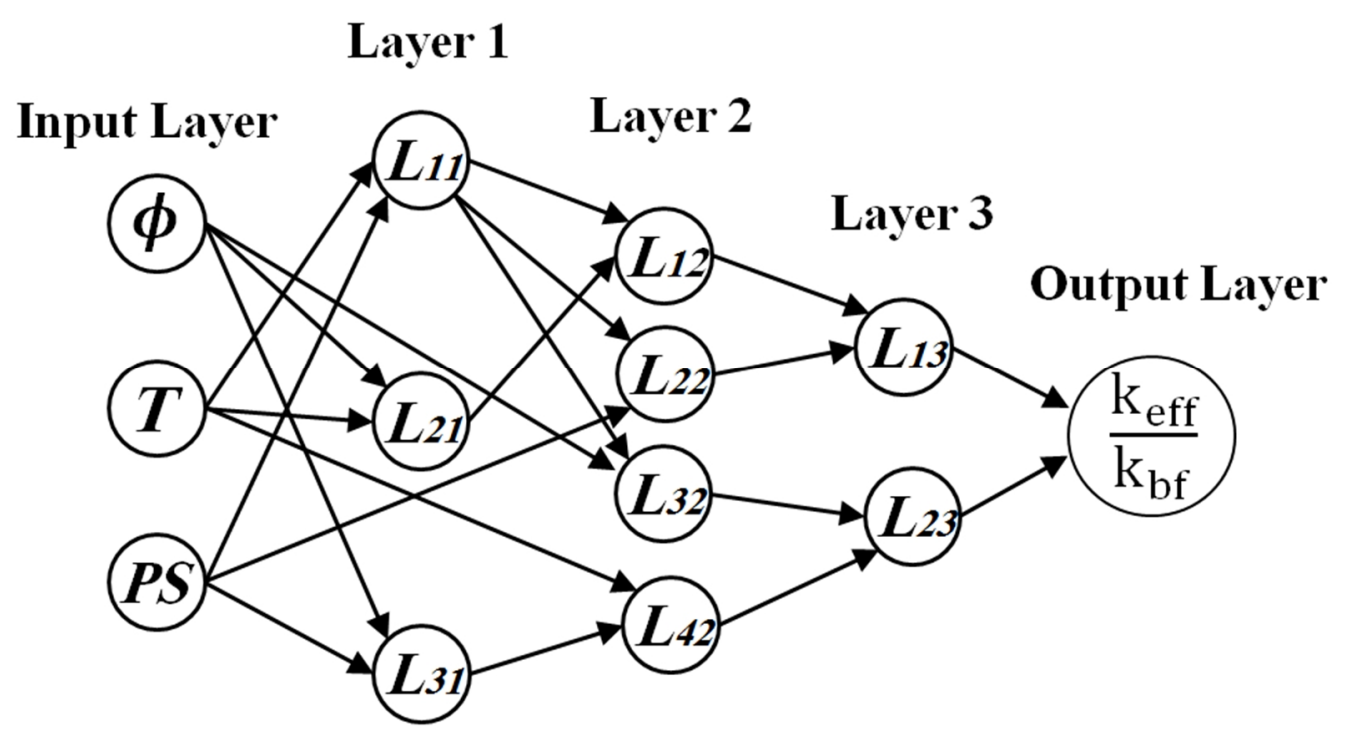

Fig.2 Structure of GA-PNN-type neural network for thermal conductivity ratio $\left(\mathrm{k}_{\mathrm{eff}} / \mathrm{k}_{\mathrm{bf}}\right)$ modelling

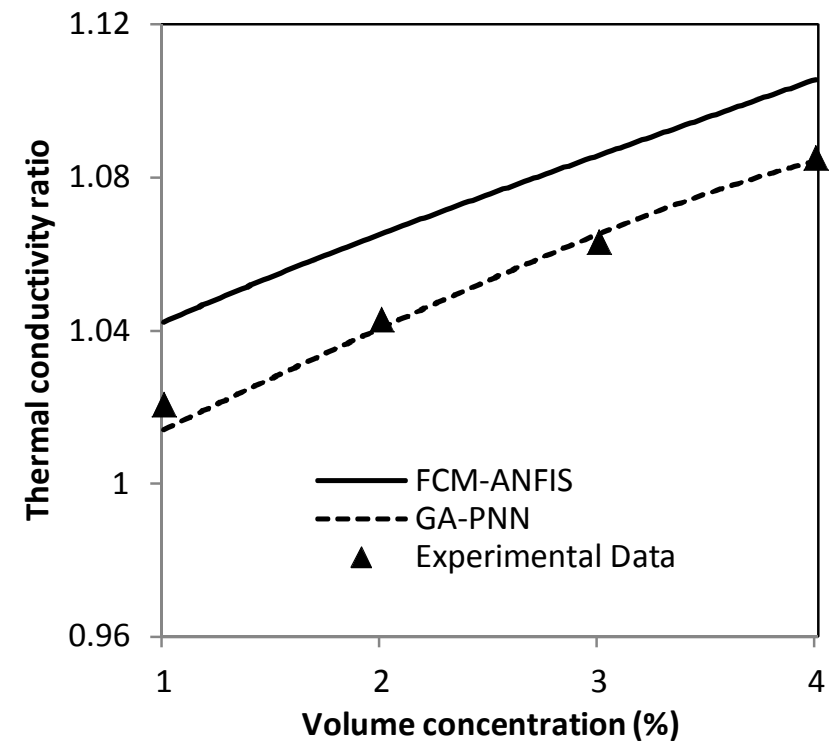

Fig.3 Comparison between the experimental data of Lee et al. [27] and the proposed models for $P S=38.4 \mathrm{~nm}$ and $T=21^{\circ} \mathrm{C}$ 


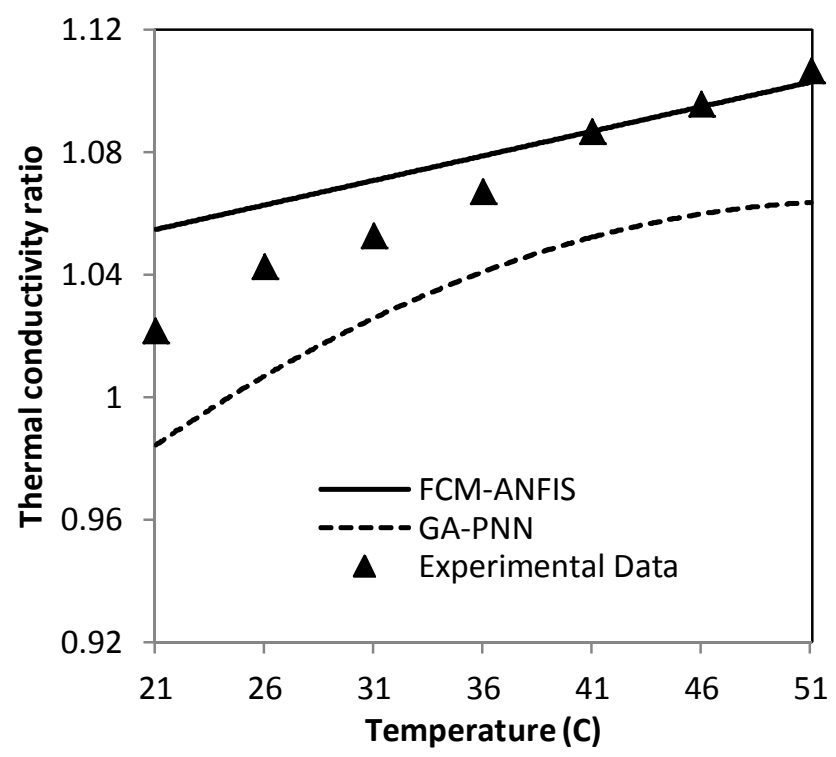

Fig.4 Comparison between the experimental data of Putra et al. [26] and the proposed models for $P S=131.2 \mathrm{~nm}$ and $\phi=1 \%$

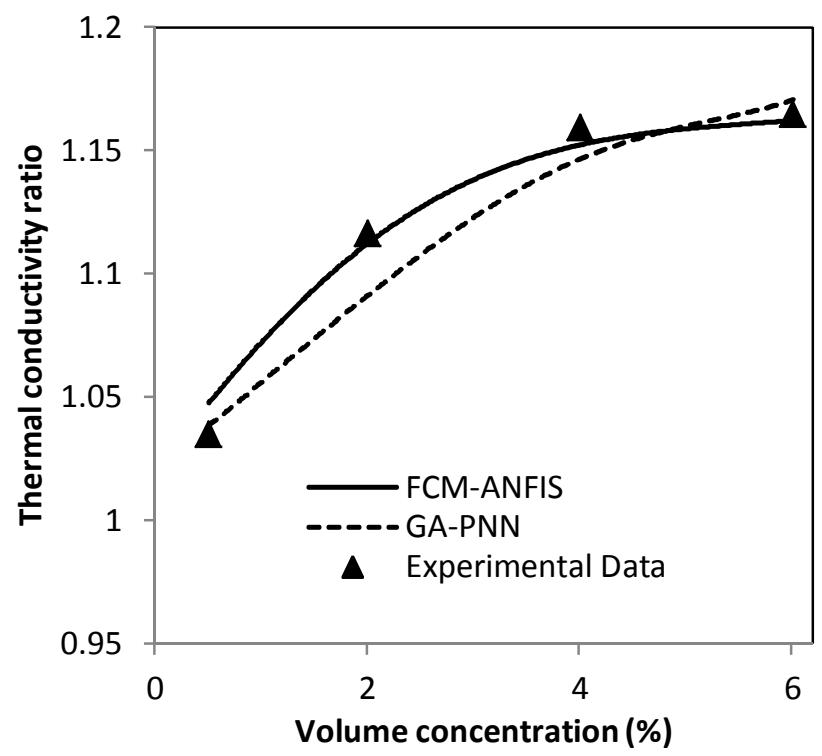

Fig.5 Comparison between the experimental data of Li and Peterson [22] and the proposed models for $P S=36 \mathrm{~nm}$ and $T=30.5^{\circ} \mathrm{C}$ 


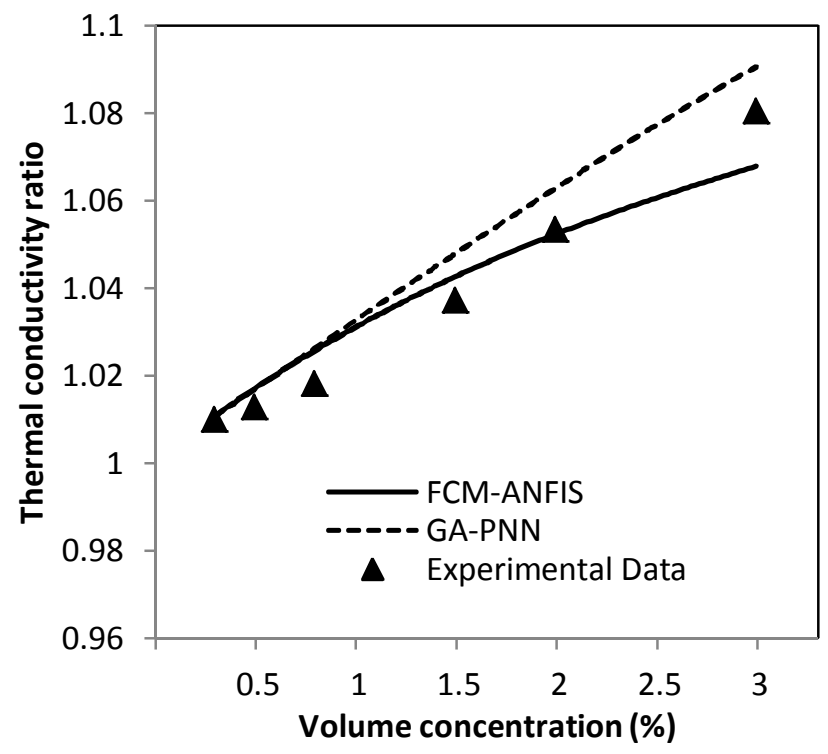

Fig.6 Comparison between the experimental data of Kim et al. [30] and the proposed models for $P S=38 \mathrm{~nm}$ and $T=25^{\circ} \mathrm{C}$

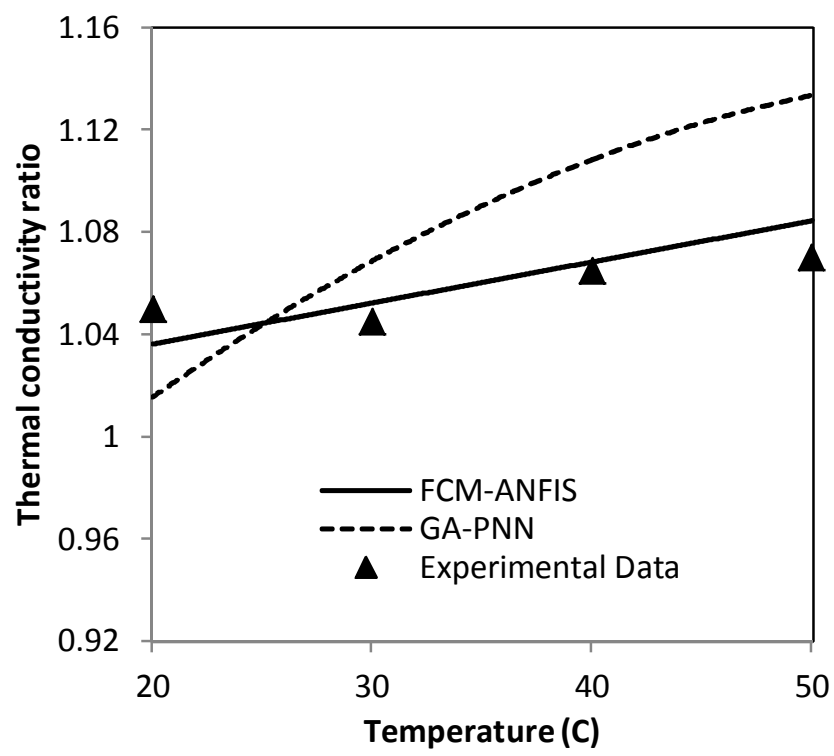

Fig.7 Comparison between the experimental data of Patal et al. [23] and the proposed models for $P S=150 \mathrm{~nm}$ and $\phi=2 \%$ 